\title{
Frequency and percolation dependence of the observed phase transition in nanostructured and doped $\mathrm{VO}_{2}$ thin films
}

\author{
Angus R. Gentle, Geoff B. Smith, and Abbas I. Maaroof \\ Institute of Nanoscale Technology, University of Technology, Sydney \\ PO Box 123, Broadway, NSW, 2007, Australia \\ g.smith@uts.edu.au
}

\begin{abstract}
The response to applied electric fields of vanadium dioxide thin films above and below the phase transition depends on the size of grains if below $\sim 200 \mathrm{~nm}$ across, and on aluminum doping above a critical concentration. $T_{c}$ drops as doping level increases, but does not depend on grain size. The observed phase transition undergoes a remarkable qualitative shift as the applied field goes from optical to low frequencies. The expected insulator to metal transition is found at optical frequencies, but at low frequencies an insulator-to-insulator transition occurs. Optical switching at both $\mathrm{T}<\mathrm{T}_{\mathrm{c}}$ and $\mathrm{T}>\mathrm{T}_{\mathrm{c}}$ is nearly independent of doping level and grain size. In contrast dc properties in both phases are sensitive to both factors. The band gaps from optical and dc data differ, and densities of states change with doping level. Such behaviour can arise if there is a transient phase change. The way doping and grain size can support such a phase is discussed. Only individual nanograins need to switch phases coherently to explain data, not the whole sample. Resistance as a function of composition across the transition was derived using effective medium compositional analysis of optical data in the hysteresis zone. The percolation thresholds are not at the usual $\mathrm{T}_{\mathrm{c}}$ values.
\end{abstract}

Keywords: vanadium dioxide, metal-insulator, phase transition, nanograin, doping, percolation

\section{INTRODUCTION}

The first order metal-insulator phase transition in undoped $\mathrm{VO}_{2}$ with large grains occurs at $T_{\mathrm{c}}$ $=340 \mathrm{~K}$. It is usually assumed to be a metal-insulator transition because the high temperature phase has a lower dc resistivity than the low temperature state by several orders of magnitude, and at near infrared (NIR) wavelengths a large rise in reflectance from low values occurs as temperature rises from $\mathrm{T}<\mathrm{T}_{\mathrm{c}}$ to $\mathrm{T}>\mathrm{T}_{\mathrm{c}}$. However it has become increasingly clear from a range of experiments in recent times [1-3] that neither phase behaves in a manner consistent with the normal optical and electrical characteristics of standard semiconductors and metals. These unusual characteristics are particularly apparent for the high temperature phase but as we will see the semiconductor phase also has unusual character. We have recently shown that in small grain thin film format the high temperature phase does not behave electrically like a metal for samples with small enough grain sizes or with any grain size when doped with aluminium $[4,5]$. Instead its conductivity continues to be thermally activated. It does however behave optically like a plasmonic metal. That is at NIR frequencies the properties at $T>T_{c}$ are those of a Drude metal, while at low enough frequencies a residual band gap is apparent. Such behaviour is traditionally incompatible. The critical factors found from experiment so far that influence the strength of this residual gap will be outlined and we will consider the impact of doping in more detail to better understand the way electronic properties change on doping. The density of states near the Fermi level at low frequencies is of particular interest. A variety 
of band gaps appear at low frequencies but at high frequencies the band structure appears to be relatively stable. To shed further light on this puzzling behaviour the link between the percolation transition for each component and $\mathrm{T}_{\mathrm{c}}$ will be examined.

In the semiconducting state $\mathrm{Al}$ doping causes resistivity to drop, as does $\mathrm{W}$ doping $[1,4,6]$. Raising carrier density lowers the transition temperature, and this has been the main technical motivation for doping, namely to push $\mathrm{T}_{\mathrm{c}}$ closer to room temperature. Our data that follows on concentration dependence shows clearly that doping does not occur via the traditional impurity ionization route. It is the ability of electron pairs on two neighbouring $\mathrm{V}^{4+}$ ions, as shown in Fig. 1, to locally correlate their motion, that leads to the creation of stable singlet dimers and a band gap [7]. Various states that might form in the metal state based on these pairs are also shown in Fig. 1.

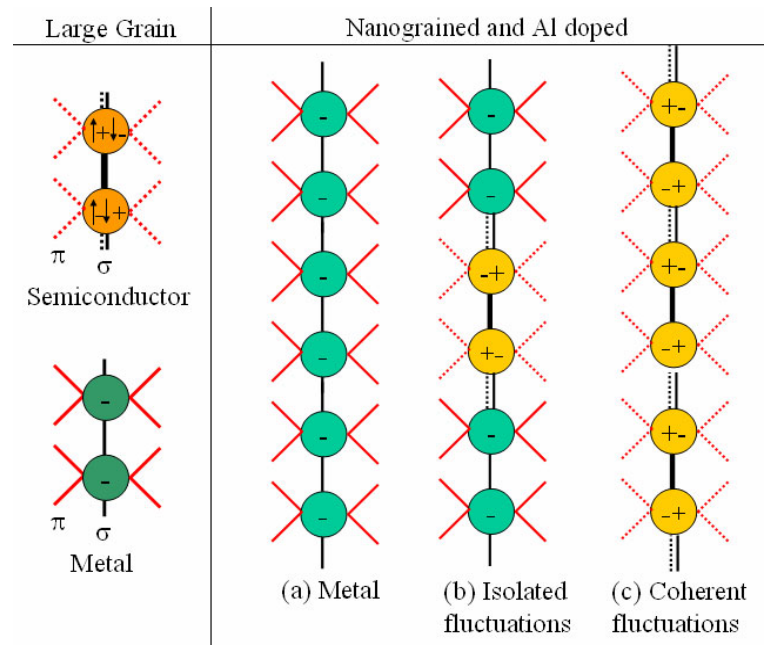

Fig. 1. Dimer singlet pairs in each $\mathrm{VO}_{2}$ phase and states that may form from such pairs in nanograin and $\mathrm{Al}$ doped samples [4]. $\pi$-bonds are empty in the semiconductor phase, as indicated by broken lines. Only four of eight $\pi$-bonds to oxygen are shown for simplicity. "Coherent fluctuations" shown are for the metal state, but may also occur below $\mathrm{T}_{\mathrm{c}}$.

This correlation produces a bonding state, plus an energy gap to the anti-bonding states. The local correlation is believed to be broken if a hole is produced at any dimer as this allows the other carrier to move and also produces a local lattice distortion [3]. A complete phase transition occurs if hole density or density of excited dimers reaches a critical level believed to be $\sim 0.018 \%$ [8]. The ability to induce the transition by doping, raising temperature, and band bending with an external field all involve raising hole density. Our aim here is to explore the way doping and grain size lead to these frequency dependencies and unusual dynamics.

\section{BRIEF DESCRIPTION OF VO, PROPERTIES}

\subsection{Conductivity of $\mathrm{VO}_{2}$}

Lattice dynamics and conductivity in the $\mathrm{THz}$ range are able to restore correlations [3] in pump-probe experiments, provided total photon fluence in the pump pulses is not above a critical value. However if sufficient density of local holes are produced a meta-stable lattice- 
electronic system arises in which de-localisation of carriers occurs over an extended time. These ideas need further development to explain why in doped and nano-gain samples, there is persistence above Tc of correlation dynamics in dc conductivity, but not at optical frequencies. We will see that doping and nanograin sizes have a weak impact at $T>T_{c}$ on the (Drude) plasma frequency $\omega_{\mathrm{P}}$, but a strong impact on apparent carrier densities in dc measurements. Normally for metals the two carrier densities $n_{c}$ are the same, since in a Drude metal $\omega_{P}^{2}=\frac{n_{c} e^{2}}{\varepsilon_{o} m^{*}}$ with $m^{*}$ carrier effective mass, $\varepsilon_{0}$ the permittivity of free space, and $e$ electronic charge. Thus the resistivity $\rho$ can be rewritten in terms of plasma frequency since

$$
\frac{1}{\rho}=\frac{n_{c} e^{2} 2 \pi}{\omega_{\tau} m^{*}}=\frac{\omega_{P}^{2} \varepsilon_{o} 2 \pi}{\omega_{\tau}}
$$

with $\omega_{\tau}=2 \pi / \tau$ and $\tau$ the relaxation time for carrier scattering. Using optically derived parameters in Eq. (1) to estimate de resistivity gives close values to data for most normal metals apart from small variations in the magnitudes of $\omega_{\tau}$ between optical frequencies and dc. This is because $n_{c}$ and $m^{*}$ are usually almost the same in both data sets. The linear temperature coefficient of $\rho$ normally comes from $\omega_{\tau}(\mathrm{T})$. Unusual changes in $m *$ in this nonFermi liquid system do not seem to explain observed changes with frequency.

Recent theoretical developments in the theory of strongly correlated conductors and unusual superconductors are relevant $[9,10]$. One of their major thrusts is to develop a better understanding of the unusual metallic state as a pre-requisite to understanding the coherent superconductor state that may arise in some strongly correlated compound conductors. One phase that can arise in these systems is called a "bad metal" because the carrier mean free path is less than a lattice constant and charge transport is then incoherent. $\mathrm{VO}_{2}$ at $\mathrm{T}>\mathrm{T}_{\mathrm{c}}$ is a "bad metal" as it does satisfy this criteria. Its anomalously high relaxation rate $\omega_{\tau}$ is of order $0.7 \mathrm{eV}$ $[1,5,13]$. Normal Drude behaviour is not expected in "bad metals" but our samples are clearly Drude like at high frequency. "Bad metals" do have negative temperature coefficients of resistance, but not the simple thermally activated behaviour we will demonstrate. The proposed phase diagrams $[9,10]$ for these systems have an intermediate regime of normal Fermi liquid or metal, before the onset of bad metal behaviour at a higher temperature. In contrast we find a direct transition from the non-magnetic insulator to the unusual dual metal/insulator state.

\subsection{Percolation and the metal-insulator phase transition}

As the $\mathrm{VO}_{2}$ phase transition has a wide hysteresis zone which consists of a metal-insulator mix the transition can be considered to be a percolative phase transition. Two component nanostructured networks with one phase metallic and the other insulating undergo a metalinsulator transition (MIT) at the critical concentration of metal known as the percolation threshold, where the highly conducting phase first forms a connected network. Typically DC resistivity $\rho$ changes by several orders of magnitude and there are significant qualitative changes in optical response in the wavelength range where the dense metal phase would begin to reflect strongly. Experimentally in the hysteresis zone of $\mathrm{VO}_{2}$ the optical response appears to be homogeneous and specular in thin films. Thus effective medium approaches can be used to model both optical and dc data in the mixed phase region, implying also that the individual components of the mixed phase are nanoscale. There is however an important difference between the thermal description and percolation description of such a transition. The thermal transition is usually taken to be at the temperature when there are equal quantities of each 
phase while the percolation transition occurs when the emerging conducting phase first forms a long range linked network. Let $\mathrm{f}_{\mathrm{m}}$ be the metal fill factor. An interconnected network forms at a critical volume fraction of conductor where $f_{m}=f_{c}$ which may occur at a temperature which differs from the thermal critical temperature $T_{c}$. $T_{c}$ is thus traditionally taken to be the temperature where either the resistivity $\rho$ or the optical reflectance is half way between the two extremes found in a pure insulator and pure metal. The correlation between the two transitions has not been explored previously with any rigor.

For an effective medium treatment in the optical domain we use here the Bruggeman model for effective dielectric constant $\varepsilon^{*}$ expressed at each wavelength in terms of an effective depolarisation factor $\mathrm{L}$, metal volume fraction $\mathrm{f}_{\mathrm{m}}$ and metal and insulator dielectric constants $\varepsilon_{\mathrm{m}}$ and $\varepsilon_{\mathrm{i}}$ as

$$
f_{m}\left[\frac{\varepsilon_{m}-\varepsilon^{*}}{\varepsilon^{*}+L\left(\varepsilon_{m}-\varepsilon^{*}\right)}\right]+\left(1-f_{m}\right)\left[\frac{\varepsilon_{i}-\varepsilon^{*}}{\varepsilon^{*}+L\left(\varepsilon_{i}-\varepsilon^{*}\right)}\right]=0
$$

The critical volume fraction in this model occurs at $f_{m}=f_{c}=L$. Plasmonic response also vanishes at this point and the effective or homogenized plasma frequency falls as $f_{m}$ approaches $f_{c}[16,17]$. In 3-D with optical isotropy, we expect $L=1 / 3$ which also implies a single percolation $f_{c}$ value of $0.33[16,18]$. Fitting normal incidence optical spectra in the hysteresis zone can supply the relevant $L$ value and the various $f_{m}$ assuming topography is constant. Note however that if each component phase in the effective medium were to span the film thickness the structure is 2-dimensional and $2 \mathrm{D}$ isotropy requires that $\mathrm{L}=\mathrm{f}_{\mathrm{c}}=0.5$. For other $\mathrm{f}_{\mathrm{c}}$ values optical anisotropy is expected.

Turning to de data, one simply exchanges conductivity $\sigma$ for $\varepsilon$ in Eq. (2). If $\sigma_{I}$ is sufficiently small relative to $\sigma_{m}$ that it can be neglected, one can write (to a good approximation when the metal phase dominates)

$$
\sigma^{*}=\sigma_{m} \frac{f_{m}-L}{1-L}
$$

$\mathrm{f}_{\mathrm{c}}$ clearly equals $\mathrm{L}$ in this approximation. However in percolation problems it is usual to find experimentally that resistance approaches criticality exponentially with critical exponents $\alpha_{ \pm}$ near $f_{c}$ and the sign of $\alpha$ determines if above or below the transition, such that $R=A_{ \pm}\left|f_{m}-f_{c}\right|^{ \pm \alpha_{ \pm}}$. $\mathrm{A}_{ \pm}$are constants $[18,20]$. If this model is used at all $\mathrm{f}$ then at $f_{m}=1$ we must set $A_{+}=R_{A u}\left(1-f_{c}\right)^{\alpha_{+}}$. We can thus pinpoint $\mathrm{f}_{\mathrm{c}}$ by the $\mathrm{f}_{\mathrm{m}}$ value where $\mathrm{R}$ versus $\mathrm{f}_{\mathrm{m}}$ changes most rapidly. A phenomenological effective medium model has been developed for all $\mathrm{f}_{\mathrm{m}}$ and $\sigma_{\mathrm{i}}$ including these exponents $[21,22]$ which works quite well for many mixtures at low frequency but has yet to be tried optically (i.e. replacing $\varepsilon$ for $\sigma$ ). It is given by

$$
f_{m}\left[\frac{\sigma_{m}^{1 / \alpha_{+}}-\sigma^{*^{1 / \alpha_{+}}}}{\sigma_{m}^{1 / \alpha_{+}}+(d-1) \sigma^{* 1 / \alpha_{+}}}\right]+\left(1-f_{m}\right)\left[\frac{\sigma_{i}^{1 / \alpha_{-}}-\sigma^{*^{1 / \alpha_{-}}}}{\sigma_{i}^{1 / \alpha_{-}}+(d-1) \sigma^{* 1 / \alpha_{-}}}\right]=0
$$


with $\mathrm{d}$ being the dimensionality of the structure which from equations $(2,3)$ is expected to equal $1 / \mathrm{L}$.

\subsection{Band gaps, density of states and response functions}

Optical and resistance data has to be processed to extract the parameters that tell us what is happening to the band structure and provide insights into the nature of the "metal" phase. Spectral transmittance and reflectance at normal incidence from $350 \mathrm{~nm}$ to $2,500 \mathrm{~nm}$, in combination with film thickness enables the complex refractive index $\mathrm{N}(\omega)=(\mathrm{n}, \mathrm{k})$, and the relative dielectric constant $\varepsilon(\omega)=\mathrm{N}^{2}$, to be established at each frequency $\omega$. For $\mathrm{VO}_{2}$ the Lorentz-Drude (LD) oscillator expression

$$
\varepsilon(\omega)=\varepsilon_{\infty}+\sum_{j} \frac{A_{j}}{\omega_{j}^{2}-\omega\left(\omega+\omega_{\tau, j}\right)}-\frac{\omega_{p}^{2}}{\omega\left(\omega+i \omega_{\tau}\right)}
$$

gives an excellent fit to the optical constants. The last term is the Drude contribution and is not present in $\mathrm{VO}_{2}$ at $\mathrm{T}<\mathrm{T}_{\mathrm{c}}$. The second term gives the inter-band contributions centred at frequency $\omega_{\mathrm{j}}$ with joint bandwidth $\omega_{\tau, \mathrm{j}}$. $\mathrm{A}_{\mathrm{j}}$ gives the spectral weight of each transition, and depends on the joint density of states. $\varepsilon_{\infty}$ is a real constant containing effects of any high frequency oscillators not included explicitly. In the un-doped semiconductor phase the smallest value of $\omega_{\mathrm{j}}$ with $\mathrm{j}=1$, should normally be close to the smallest band gap (in $k$-space) between the valence and conduction band, and also match the gap found from the thermal activation energy $\Delta \mathrm{E}_{\mathrm{sc}}(\mathrm{c}, \mathrm{g})$ when concentration $\mathrm{c}$ of aluminium dopant atoms equals zero in expression (6) for the semiconductor's dc resistivity $\rho_{\mathrm{sc}}$. Our finding of sensitivity of band gap to grain size $g$ accounts for the explicit inclusion of symbol $g$. We thus then expect $\omega_{1} \sim E_{g}$ the band gap, using $\mathrm{E}_{\mathrm{g}}=2 \Delta \mathrm{E}_{\mathrm{sc}}(0, \mathrm{~g})$. Data indicates this relation does hold, but only for large grain samples with grain size $\mathrm{g} \sim 300 \mathrm{~nm}$ or larger. In general however we have found that

$$
\rho_{s c}(T, c, g)=\rho_{0, s c}(c, g) e^{\left[\Delta E_{s c}(c, g) / k T\right]}
$$

where $\mathrm{c}$ is the concentration of aluminium dopant atoms and the prefactor $\rho_{0, \mathrm{sc}}$ depends on relaxation frequency and the joint density of states (from the top of the valence band and bottom of the conduction band). $\rho_{0, s c}$ has a weak $T$ dependence which is not shown explicitly because the thermal activation energy dominates this aspect of response. Expression (6) looks normal but has very unconventional features. It is based on a careful analysis of data, including the wide variations we previously reported [4] for activation energies with changes in both $\mathrm{g}$ and $\mathrm{c}$, plus some new data. A novel plot summarising semiconductor gaps in several sample types appears in section 3. $\rho_{0, \mathrm{sc}}$ is also found to be quite variable as $\mathrm{g}$ and $\mathrm{c}$ change. One surprise is that while smaller $g$ is conventionally expected in both states to raise resistivity via an increased relaxation rate, it does not. Instead $\rho$ decreases significantly as $g$ gets smaller [4,1]. We shall see a fall in densities of states near the band edges is outweighed by a fall in band gap and hence activation energy, to cause this unexpected impact for dc fields. Optical data should change if the band gap falls as $g$ falls but does not. The impact of doping level $\mathrm{c}$ also needs to be distinguished from normal semiconductor doping where easy ionization of weakly bound electrons or holes on dopants means that thermally activated dc response is not significant at room temperature. $\mathrm{In}_{\mathrm{VO}_{2}}$ : $\mathrm{Al}$ and $\mathrm{VO}_{2}: \mathrm{W}$ in contrast, thermally activated semiconductor response is still dominant. Deep, less easily ionized gap states are a possible explanation, but more likely are moderate falls in the gap itself and rises in densities 
of states with increase in $\mathrm{c}$ as found with small $\mathrm{g}$. This impact of doping on band gap should also show up in optical data but does not. Any significant density of doping related deep gap states would show up optically via additional lower energy transitions than the $\omega_{1}$ values found in undoped samples, but no such peaks arise. Thus despite outward appearances of normal dc and optical response the semiconductor state in nano-grain and doped $\mathrm{VO}_{2}$, when this data is considered carefully and in combination, is not that of a normal semiconductor. The main puzzle is the apparent differences in semiconductor band structure as seen with optical and dc fields.

The state at $\mathrm{T}>\mathrm{T}_{\mathrm{c}}$, usually treated as a metallic state, is more obviously anomalous than the semiconductor state. Not only does it have "bad metal" like super-high relaxation rates, but its optical and dc responses for small enough g, and any doping level $\mathrm{c}$ at all grain sizes, represent qualitatively distinct physical states. One is like a metal and the other like an insulator. The optical data, as in the examples in Figs. 2 and 3, is unequivocally plasmonic or Drude like for wavelengths longer than $1 \mu \mathrm{m}$. Resistivity however is that of a semiconductor, but not a normal semiconductor. This is given in Eq. (7), using similar notation to Eq. (3) except for the subscript $m$ for "metal" phase, as

$\rho_{m}(T, c, g)=\rho_{0, m}(c, g) e^{\left[\Delta E_{m}(c, g) / k T\right]}$.

The dependencies on $\mathrm{g}$ and $\mathrm{c}$ discussed above for the semiconductor are mirrored in the $\mathrm{dc}$ response at $\mathrm{T}>\mathrm{T}_{\mathrm{c}}$, though the activation energy $\Delta \mathrm{E}_{\mathrm{m}}(\mathrm{c}, \mathrm{g})<\Delta \mathrm{E}_{\mathrm{sc}}(\mathrm{c}, \mathrm{g})$ in all samples studied to date. It appears that finite $\Delta \mathrm{E}_{\mathrm{m}}(\mathrm{c}, \mathrm{g})$ is not possible for grain average sizes over $300 \mathrm{~nm}$ in the absence of doping.

\section{EXPERIMENT AND RESULTS}

\subsection{Film preparation and nanostructure}

Vanadium thin films were first prepared by de magnetron sputtering onto optically polished super-white glass and mica substrates. The base pressure was less than $10^{-6}$ Torr, while sputtering was carried out in the presence of flowing argon, at a pressure of $2 \times 10^{-3}$ Torr. To ensure good homogeneity and crystallinity, a $450{ }^{\circ} \mathrm{C}$ substrate temperature was used during deposition. After deposition, the films were converted into $\mathrm{VO}_{2}$ by controlled oxidization. Air was introduced at various pressures of 0.1 Torr to 1 Torr and the substrate temperature was held between $400{ }^{\circ} \mathrm{C}$ and $450{ }^{\circ} \mathrm{C}$ for 5 hours. Film thicknesses ranged from $20 \mathrm{~nm}$ to $150 \mathrm{~nm}$, with the annealing time varied to suit. For vanadium thicknesses of $30 \mathrm{~nm}$ to $100 \mathrm{~nm}$ annealed at $450{ }^{\circ} \mathrm{C}$ the required annealing time was given by $t$ (hours $)=3.622+0.047 *$ thickness $(\mathrm{nm})$. Most films produced on glass substrates had an average grain size of approximately $50 \mathrm{~nm}$ while the mica substrate produced $100 \mathrm{~nm}$ grain sizes. It was also possible to alter the grain size via varied deposition and annealing temperatures from $40 \mathrm{~nm}$ to $60 \mathrm{~nm}$ on a glass substrate. Grain sizes were determined by high resolution imaging and example structures can be seen in Ref. 4.

\subsection{Optical and resistance data}

The DC electrical properties of $\mathrm{VO}_{2}$ films were measured using the "ohms-per-square" method with a four-wire resistance measurement and evaporated electrodes arranged for electrically sampling a square surface area of the sample. Optical measurements of spectral transmittance $\mathrm{T}(\lambda)$ at normal incidence and spectral reflectance $\mathrm{R}(\lambda)$ at $8^{\circ}$ off-normal incidence, were carried out using a Cary 5E UV-VIS-NIR spectrophotometer in the UV, 
visible and near-infrared over the wavelength range $2500 \mathrm{~nm} \geq \lambda \geq 300 \mathrm{~nm}$. Sample heating was carried out with a purpose-built heater system designed for the spectrophotometer and temperature was monitored by a thermocouple attached directly to the film surface. The spectra were measured at $25{ }^{\circ} \mathrm{C}$ and $80{ }^{\circ} \mathrm{C}$, for $100 \%$ semiconductor and metallic phases respectively. A spectrum above $100{ }^{\circ} \mathrm{C}$ was also taken to ensure no unusual temperature related changes occurred as temperature rose. Unlike the resistivity data no change was observed. Optical hysteresis behaviour at the transition was studied by recording NIR transmittance at a wavelength of 2,000 nm for every $1{ }^{\circ} \mathrm{C}$ change in temperature. $2,000 \mathrm{~nm}$ was chosen as a wavelength in the NIR. As can be seen in the spectra in Fig. 2, for a $50 \mathrm{~nm}$ grain size there is a large transmittance switch at 2,000 nm. These spectra are shown in Fig. 2 fitted with the oscillator models of Eq. (5). Spectra for doped films are quite similar but have different $T_{c}$ values.

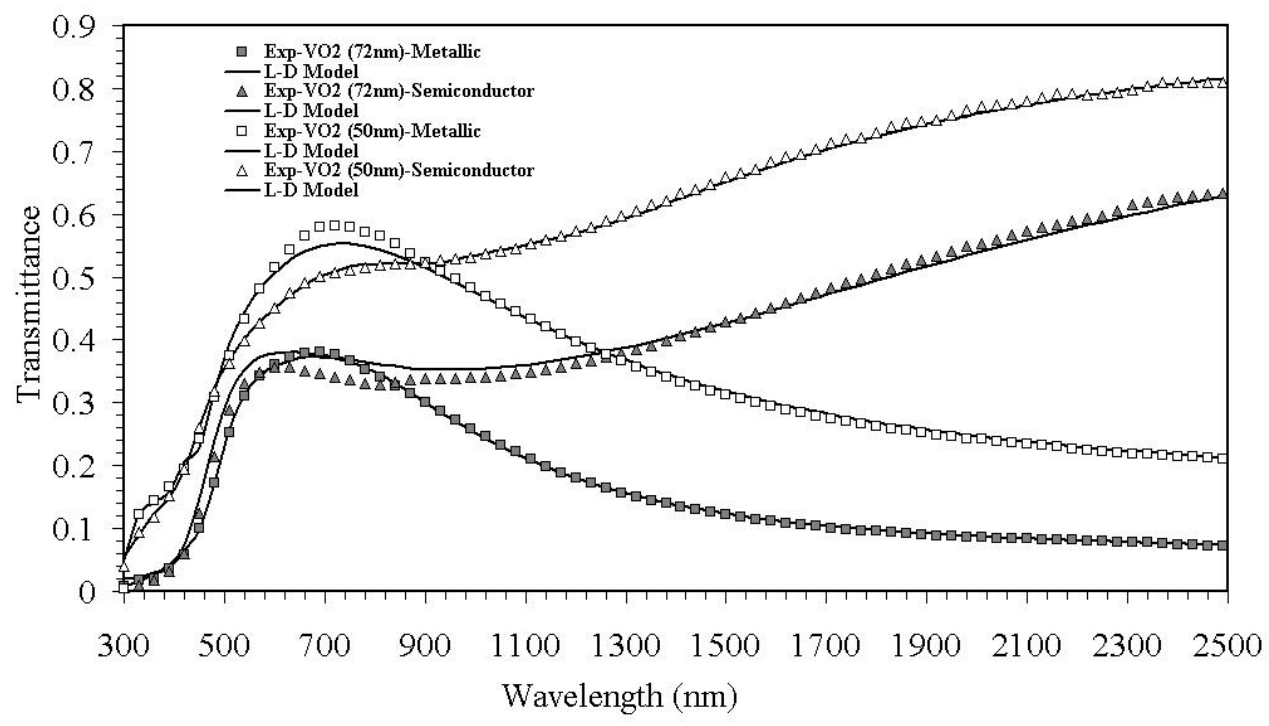

Fig. 2. Spectral transmittance for two VO2 films on glass with $50 \mathrm{~nm}$ grain sizes. Continuous curves are best fits of this and corresponding reflectance data with a Lorentz-Drude oscillator model, which yields each film's optical constants.

Complex refractive indices (n, k) obtained from the LD fits using Eq. (5) to these $\mathrm{R}$ and $\mathrm{T}$ spectra show clearly in Fig. 3 at $80{ }^{\circ} \mathrm{C}$ the plasmonic switchover to $\mathrm{k}>\mathrm{n}$ occurs near $1 \mu \mathrm{m}$ in the metal phase. $\mathrm{n}$ and $\mathrm{k}$ for wavelengths below $0.5 \mu \mathrm{m}$ show very little change at the phase transition, with an inter-band transition around $2.8 \mathrm{eV}$ common to both phases. This indicates the main impact of the strong electron-electron Coulomb repulsion $U$ or Hubbard interaction, is preserved. These $\mathrm{n}, \mathrm{k}$ characteristics are quite close to those for single crystals [11], that is grain size has only a small impact optically. The impact of the transitions across the semiconductor band gap on $\mathrm{n}, \mathrm{k}$ can be seen in the low peak between $900 \mathrm{~nm}$ to $1400 \mathrm{~nm}(1.4$ $\mathrm{eV}$ to $0.8 \mathrm{eV}$ ) in the semiconductor's extinction coefficient $\mathrm{k}$. This peak cannot be attributed to the plasmonic resonant response of a residual "metal" phase inclusion in the insulator based on the indices at these wavelengths. These transitions are the ones we expect to match from temperature dependence of dc electrical data. That is not the case for this film but is so for large grains. An overview of the way band gaps either side of $T_{c}$ relate to each other in the optical and de domains can be seen in Fig. 4 in which data points are designated by grain size 


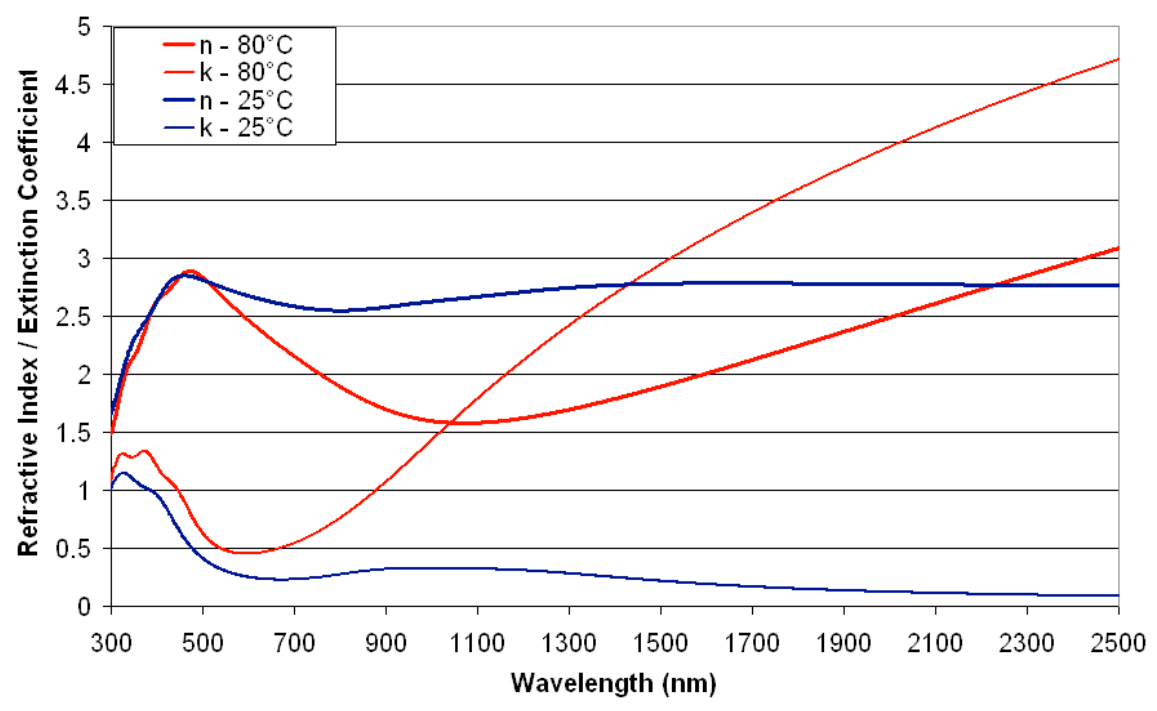

Fig. 3. Complex refractive indices from $300 \mathrm{~nm}$ to $2500 \mathrm{~nm}$ of undoped $\mathrm{VO}_{2}$ thin film with $50 \mathrm{~nm}$ grain sizes in the insulator and metal phases.

and doping level. All de data is for grain sizes of $100 \mathrm{~nm}$ or less and thus shows a finite gap at $\mathrm{T}>\mathrm{T}_{\mathrm{c}}$. Increased doping always increases the $\mathrm{dc}$ gaps in the high $\mathrm{T}$ phase but the semiconductor dc gap at $\mathrm{T}<\mathrm{T}_{\mathrm{c}}$ seems to pass through a minimum around $0.3 \mathrm{eV}$. This is for an undoped film with $50 \mathrm{~nm}$ average grain size. It is possible that even smaller grain size might eliminate the transition in dc resistance, which would be interesting if as here the full optical transition persists. In general as $g$ falls and $\mathrm{c}$ rises, we find changes in $\rho_{0, \mathrm{sc}}$ in all samples. The net result is however dominated by a fall in $\Delta \mathrm{E}_{\mathrm{sc}}$ which leads to an overall anomalous drop in resistivity.

As an example of the changes with doping single crystals; after analysis of data in Ref. 12 we find: undoped, $\rho_{0, \mathrm{sc}}=5.5 \times 10^{-7} \Omega \mathrm{cm}$; doped with $0.247 \% \mathrm{Al}, \rho_{0, \mathrm{sc}}=1.1 \times 10^{-4} \Omega \mathrm{cm}$, and doped with $0.49 \% \mathrm{Al}, \rho_{0, \mathrm{sc}}=8.4 \times 10^{-3} \Omega \mathrm{cm}$. This implies densities of states near the gap are decreasing with doping. Changing our film grain size from $100 \mathrm{~nm}$ to $50 \mathrm{~nm}$ raises $\rho_{0, \mathrm{sc}}$ from $2.4 \times 10^{-5} \Omega$ cm to $2.4 \times 10^{-3} \Omega$ cm but $\Delta \mathrm{E}_{\mathrm{sc}}$ falls from $0.304 \mathrm{eV}$ to $0.164 \mathrm{eV}$ for a net drop in $\rho$. Thus the apparent fall in state densities near the gap is swamped by the reduction in band gap and the large change in $\rho$ with grain size is unrelated to increased scattering from grain boundaries. The density of states at band gap edges and the gap itself are both changing. Relaxation rate is also far too high to start with for extra scattering to be an influence. Similar large changes occur in $\rho_{0, m}$ for various doping levels in single crystals and various grain sizesin our thin films. Optical data should show up equivalent changes in band structure, but instead indicates a near constant band structure. 


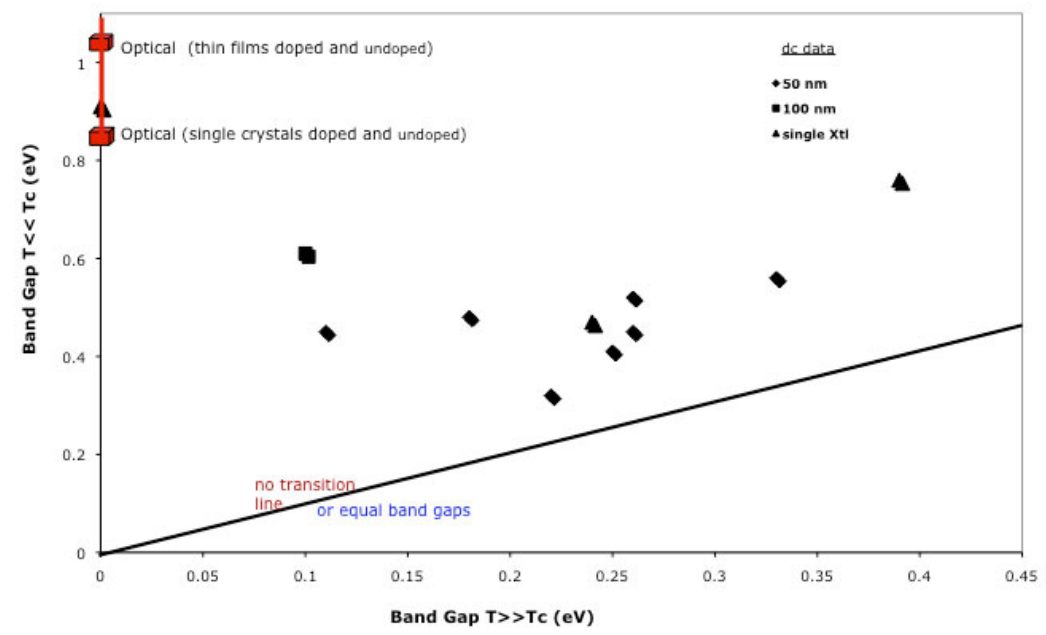

Fig. 4. The link between semiconductor band gaps at $\mathrm{T}<<\mathrm{T}_{\mathrm{c}}$ and band gaps at $\mathrm{T}>\mathrm{T}_{\mathrm{c}}$ as observed in the optical and dc domains for $\mathrm{Al}$ doped and un-doped samples. The high $\mathrm{T}_{\mathrm{c}}$ gap vanishes in all optical data, with a range of optical gaps.

Figure 5 exemplifies large differences in resistivity behaviour at the onset of hysteresis and in the high $T$ phase for two undoped samples with $50 \mathrm{~nm}$ and $100 \mathrm{~nm}$ average grain sizes. Both have thermally activated behaviour at high $\mathrm{T}$, but the $50 \mathrm{~nm}$ grain size film clearly has a

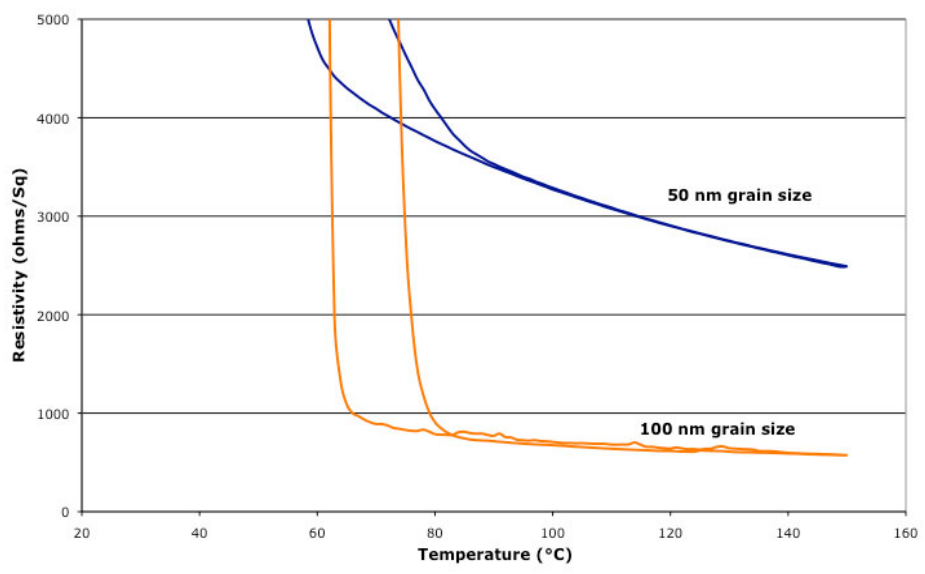

Fig. 5. Comparison of high $\mathrm{T}$, non-metallic temperature dependence of resistivity and the onset of hysteresis for two un-doped $\mathrm{VO}_{2}$ films with different grain sizes.

larger activation energy than that of the $100 \mathrm{~nm}$ grains. Optical response in contrast shows quite small differences.

\subsection{Hysteresis and the percolation transition}

We present here a preliminary analysis of the $\mathrm{VO}_{2}$ metal-insulator transition from a percolation perspective. The percolation transition to high dc conductivity as temperature is 
raised occurs at a critical volume concentration of metal $f_{m}=f_{c}$. It is of interest to see if (i) $f_{c}$ occurs at $T=T_{c}$ (ii) $f_{c}$ varies in the heating and cooling curves (iii) predicted network structures differ between dc and optical switching responses. Hysteresis curves for different levels of doping and grain size have qualitatively quite large differences [4] between the optical and de domains. In particular, apart from a shift in $\mathrm{T}_{\mathrm{c}}$ with doping level, optical hysteresis data at NIR wavelengths showed almost equal switching magnitudes in all samples, while the dc transition narrowed markedly in magnitude as doping level $\mathrm{c}$ increased or grain size $g$ decreased. From a network or percolation perspective we first assume dc and optical domain structures are the same at each $\mathrm{T}$, with the same critical concentrations. The possibility of two critical concentrations $f_{c 1}$ and $f_{c 2}$ needs to be considered, since in the hysteresis zone we are dealing with a metal-insulator composite in 3-dimensions, and in $3 \mathrm{~d}$ cermets two critical f's are found [14]. That is an intermediate regime can exist in which both phases percolate and may impact on detailed features in hysteresis plots. No evidence is found for this situation.

The analysis proceeded as follows. Optical data comprising complete spectra at set temperatures in the hysteresis zone was fitted with a suitable effective medium model which links to structure and composition. We used the basic Bruggeman model of Eq. (2) with n, k data for each component from metal and semiconductor samples outside the hysteresis zone at $90{ }^{\circ} \mathrm{C}$ and $25^{\circ} \mathrm{C}$. Being a mean field model it may be less accurate right at the transition [16], but reasonable for most of the transition zone. For the dc data we use the analogous model exchanging $\sigma$ for $\varepsilon$ in Eq. (2) [19]. Thus we first extract $n, k$ from optical $\mathrm{R}$ and $\mathrm{T}$ spectra as described in Sec. 2 above, then model to obtain fill factor $f_{m}$ and $L$ values at a range of temperatures in the hysteresis zone. The resultant plot of $\mathrm{f}$ as a function of $\mathrm{T}$ appears in Fig. 6. Quite good fits to spectra were obtained with one depolarisation factor $L=1 / 3$ in all samples which also implies a single percolation $f_{c}$ value of $0.33[16,18]$ and optical isotropy. Note however that if each component phase in the effective medium were to span the film thickness the structure is 2-dimensional and then $\mathrm{L}=0.5$ and $\mathrm{f}_{\mathrm{c}}=0.5$,

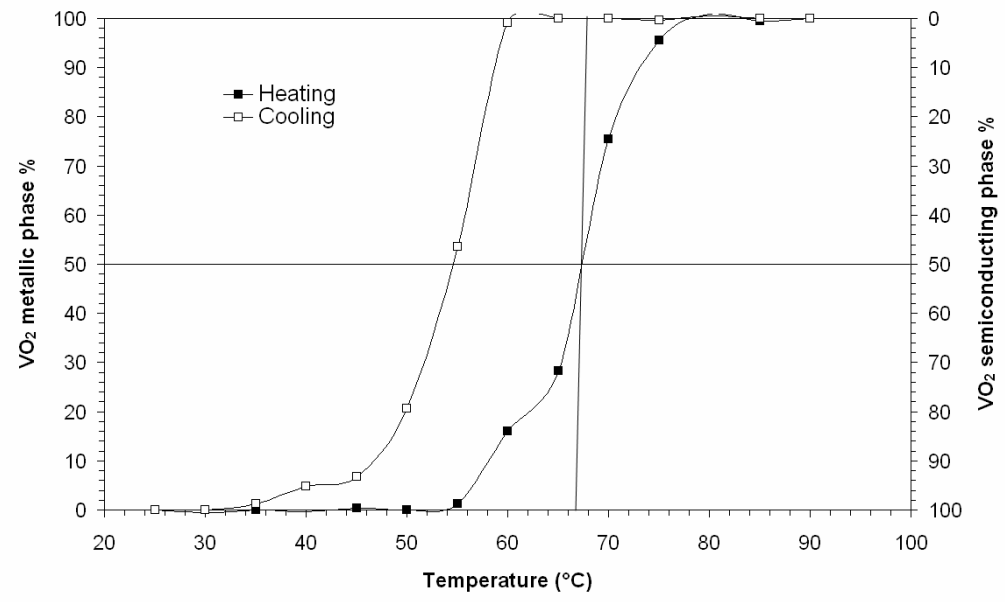

Fig. 6. Volume fractions of high $\mathrm{T}$ and semiconductor phase in the hysteresis zone as determined from optical spectra and an effective medium model. $\mathrm{f}=0.5$ is plotted horizontally for reference along with un-doped $\mathrm{T}=\mathrm{T}_{\mathrm{c}}$ also for reference.

which would occur at the $50 \%$ point in Fig. 6 , which is also where $T=T_{c}$ is usually allocated. Our finding that $\mathrm{L}=1 / 3$ would thus imply that the percolation threshold and the usual transition point are distinct. 
The $\mathrm{f}_{\mathrm{m}}$ values in Fig. 6 from optical data are mapped onto the resistivity data at each temperature to obtain a plot of $\rho$ as a function of $f_{m}$. The resulting percolation transition should show up in the heating and cooling curves in Fig. 7 at the $\mathrm{f}_{\mathrm{c}}$ value associated with $L$ $=1 / 3$ or possibly at $f_{m}=0.5$ if $d=2$. Different critical transitions are seen in Fig. 7 for the heating and cooling curves and neither occurs at $f_{m}=0.5$ or $f_{m}=0.33$. The optical model used should give one transition near the plotted vertical line, but there is no sharp change near this line. The cooling curve, which seems to be made up mainly of percolating metal phase, is more sensitive to $f_{m}$ overall than the heating curve. The comparison of heating and cooling curves is also interesting in that each has a mirrored discontinuity with the other as they approach completion at $\mathrm{f}_{\mathrm{m}} \sim 0.9$ and $\mathrm{f}_{\mathrm{m}} \sim 0.1$ respectively. Out of plane optical anisotropy would be observable if such $f_{c}$ values are occurring. Other more complex optical and EMA models such as equation (4) cannot be ruled out but require more detailed transport and optical data near $T_{c}$ to establish critical coefficients. The other issue that needs addressing is the possibility that optical and dc data across the transition actually have different $f_{c}$. This becomes possible given the contrasting transitions in optical and dc data [4].

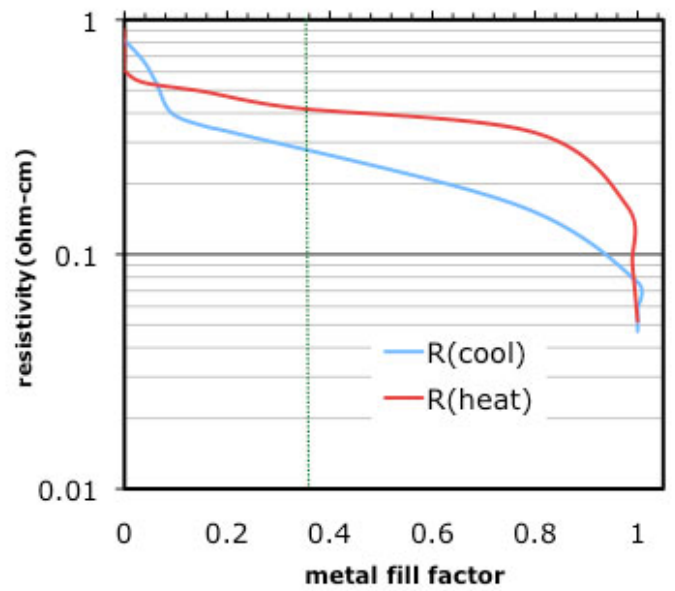

Fig. 7. Resistive percolation transition in a $50 \mathrm{~nm}$ grain size $\mathrm{VO}_{2}$ thin film using $\mathrm{f}_{\mathrm{m}}$ from optical data. The vertical line indicates where $f_{c}$ should be based on the fitting of optical data with an $L=1 / 3$ Bruggeman model.

\section{DISCUSSION}

\subsection{Transient phases and frequency dependent band structures}

Frequency dependence in apparent band gaps and associated carrier densities is unexpected and novel, even for highly correlated electronic systems. Internal dynamics with a characteristic frequency between optical values and zero must be involved. What is its nature? Normally characteristic internal dynamics, for example in the lattice or in the electronic 
system itself, would show up as an absorption band, which once traversed in probe frequency would cease to again be a major influence. To achieve a permanent shift in what is seen for all frequencies either side of some characteristic frequency implies instead a transient or time dependent phase change. If a correlated array of spin paired singlet dimers as in Fig. 1 was to switch on and off with a characteristic cycle time $t_{p}$ then the phase that is observed will depend on the applied frequency f relative to $1 / t_{p}$. For $f>>1 / t_{p}$ the paired state will not be detectable while $\mathrm{f}<<1 / \mathrm{t}_{\mathrm{p}}$ it will appear to be permanently switched on. The situation is analogous to a strobe light or image refreshment time in a display with $f$ playing the role of light sampling frequency of the brain. Two ingredients are needed to create such a situation (a) a spatially extended pairing mechanism above $T_{c}$ (b) a scattering mechanism causing decay or transience for these pairs. A transient unpaired or metal state with picosecond lifetime has been observed in pump probe experiments [3] with lifetime linked to molecular vibrations of the oxygen cage around the $\mathrm{V}^{4+}$ pairs. The critical factor in bringing about an extended relatively long lived metal state was the photon fluence, which means a sufficient density of dimers in which pairing has been destroyed. The time $t_{p}$ is in picoseconds, so we are dealing with a transition at $\mathrm{THz}$ frequencies. In our experiments it is the dopants or grain boundaries that are doing the reverse, namely allowing dimer pairs to form in sufficient density in the metal state, to enable a transient correlated array, as in Fig. 1. Local critical fluctuations can form in the metal state in strongly correlated systems. They become increasingly extended in space as one approaches a phase transition but are limited in extent by thermal scattering. The spatial limitation factor here is not thermal but the intrinsic coherence lifetime $t_{p}$ which at picoseconds is shorter than normal phonon based thermal decoherence times so it dominates. If on this timescale the dimer pairs form up with a correlation length $\xi$ which is larger than the grain size or the spacing between dopants then transient coherence and an apparent semiconductor state with lifetime $t_{p}$ may be observed. The ingredient which differs from the usual "bad metal" scenario $[9,10]$, which is temperature driven, is the role of $t_{p}$. For these ideas to work in practice it is not necessary to have each grain act in concert since the time averages will be the same well away from the critical THz frequencies, as in dc and optical data. That is though the whole sample on average behaves one way or the other, a femto or attosecond snapshot may show an admix of states, possibly matching admixes seen in the hysteresis zone. In simple terms coherence occurs only within each nanograin.

Note that transient fluctuations may also be occurring below $T_{c}$ since optical and dc gaps also differ there. The impact is less dramatic than in the high $\mathrm{T}$ phase because it involves smaller shifts, but it seems that the transient correlations can still alter energy gaps and densities of states in this phase. Fluctuations all the way to metal like bonding within the semiconductor, as noted above, occur under sufficiently intense photon pulses.

\subsection{Percolation thresholds and $T_{c}$}

Figure 6 indicates as shown that at $T=T_{c}, \mathrm{f}_{\mathrm{m}}$ is close to 0.5 . Figure 7 , however does not resemble a normal percolation plot for either $f_{c}=0.33$ or 0.50 as expected. It is more indicative of two percolation transitions in the range $0<\mathrm{f}_{\mathrm{c} 1}<0.1$ and $0.85<\mathrm{f}_{\mathrm{c} 2}<1$ since we would expect the most rapid change near $\mathrm{f}_{\mathrm{c}}$. The gradual change in $\rho$ over most $\mathrm{f}$ values is also possibly indicative of a large regime where both phases are percolating. Our analysis here is a first approximation only and needs to be repeated with two percolation transitions in 2-d and $3-\mathrm{d}$, and with the simple one threshold 2-d model with $\mathrm{L}=0.5$. All of these can be handled in a Bruggeman type model or its critical modifications [21,22]. The mechanisms noted in Sec. 4.1, which are also assumed responsible for the stark differences in the optical and dc hysteresis plots, may be at work. That is if the apparent phases in dc data were different to those in the optical domain we could explain this disparity in hysteresis. Further work, including checking the impact of some of the simplifications used in deriving Fig. 7 is needed 
to see if this is the case, or if an approach to the effective medium modeling using critical coefficients as in Eq. (4) is required.

A complete analysis of percolation in this system should include an analysis of critical percolation coefficients for $\mathrm{f}_{\mathrm{m}} \sim \mathrm{f}_{\mathrm{c}}$, to see if quasi-static homgeneous optical fits, and EMA conductivity fits to data yield the same structural parameters. The $\mathrm{T}$ dependence of the former, on evidence to date, involves only the $\mathrm{T}$ dependence of fill factors, but not of $\mathrm{n}, \mathrm{k}$ for each phase in the admix. In contrast, in the dc data the two component conductivities are both thermally activated and hence strongly $\mathrm{T}$ dependent. With both heating and cooling curves occupying a range of around $20{ }^{\circ} \mathrm{C}$ this will impact strongly on the hysteresis curves, along with the T dependence of fill factors.

One recent study [23] of component fill factors in the hysteresis zone in $\mathrm{VO}_{2}$ thin films used a 2-dimensional resistor network and compared nanocrystalline and microcrystalline transition properties using de data only. They predict plots similar to Fig. 7, but their approach has limitations. Most importantly our frequency dependent data means optical and de data are both needed to fully describe what is happening, and to validate models based on morphology. Clear evidence for thermally activated metal phase conductivity data in their nanocrystalline samples was ignored in reference [23]. Their effective medium models do not have a percolation threshold since they assumed a simple average of the two phase conductivities, which is usually only true for low $\mathrm{f}$ and high $\mathrm{f}$. Another problem is 2-dimensionality is most unlikely in a $350 \mathrm{~nm}$ thick layer with nanograins much smaller than thickness. An unusual and interesting feature of this work was a drop in $T_{c}$ with nanograins, despite the lack of doping. This may be evidence for a vanishing dc transition when grains are small enough as hinted at in our earlier work [4]. Optical data is needed to see if a normal phase transition is still apparent at high frequencies.

\section{CONCLUSION}

Compelling experimental evidence has been presented that the apparent band structures observed at optical and dc frequencies in $\mathrm{Al}$ doped $\mathrm{VO}_{2}$ are different, and that the percolation transition in resistance is not at the usual $\mathrm{T}_{\mathrm{c}}$ point when analysed using both optical and $\mathrm{dc}$ data in combination. These results are considered to be due to the existence of a localised transient or fluctuating phase transition with $\mathrm{THz}$ switching characteristics. These transients are aided by doping and by nanograin structure. This does not require the whole sample to be in one condition or the other at any instant. The individual grains can fluctuate independently between phases, such that optical fields switch much faster than the intrinsic phase switching in any one grain, while low frequency data involves many metal-insulator phase switches per field cycle. The anomalous fall in semiconductor resistance as grain size falls appears to be of similar origin to the effect of doping. A common misconception in $\mathrm{VO}_{2}$ is that dopant impact is due to normal ionized impurity doping, but here we have shown it changes the band structure.

Much further work is needed to understand these frequency dependent phases and transitions. Critical parameters such as percolation $\alpha$ and $f_{c}$, must be linked self-consistently with observed morphologies, while a full suite of optical and dc data is needed to assess the impact of nanoscale morphologies in the transition or hysteresis zone. The ideal experiment would involve snapshots taken on femtosecond or faster time scales. Nanoparticles may also have similar unusual properties.

\section{Acknowledgments}

AG thanks The Commonwealth of Australia for his APA PhD scholarship support. Ric Wuhrer and Geoff McCredie gave key support to characterisation studies and to coating work. 


\section{References}

[1] A. Gentle, A. I. Maaroof, and G. B. Smith, "Nanograin $\mathrm{VO}_{2}$ in the metal phase: a plasmonic system with falling dc resistivity as temperature rises," Nanotech.18, 025202 (2007) [doi:10.1088/0957-4484/18/2/025202].

[2] D. Brassard, S. Fourmaux, M. Jean-Jacques, J. C. Keiffer, and M. A. El Khakani, "Grain size effect on the semiconductor-metal phase transition characteristics of magnetron sputtered $\mathrm{VO}_{2}$ thin films," Appl. Phys. Lett. 87, 051910 (2005) [doi:10.1063/1.20011].

[3] C. Kuebler, H. Ehrke, R. Huber, R. Lopez, A. Halabica, R. F. Haglund Jr., and A. Leitenstorfer, "Coherent structural dynamics and electronic correlations during an ultrafast insulator-to-netal phase transition in $\mathrm{VO}_{2}$," Phys. Rev. Lett. 99, 116401 (2007) [doi:10.1103/PhysRevLett.99.116401].

[4] A. Gentle and G. B. Smith, "Dual metal-insulator and insulator-insulator switching in nanoscale and $\mathrm{Al}$ doped $\mathrm{VO}_{2}, "$ J. Phys. D: Appl. Phys. 41, 015402 (2008) [doi:10.1088/0022-3727/41/1/015402].

[5] A. Gentle, A. I. Maaroof, and G. B. Smith, "Temperature dependence of optical and transport properties in $\mathrm{VO}_{2}$ with high temperature anomalies," Curr. Appl. Phys. 8, 229-232 (2007) [doi:10.1016/j.cap.2007.10.006].

[6] A. Romanyuk, R. Steiner, L. Marot, and P. Oelhafen, "Temperature-induced metalsemiconductor transition in $\mathrm{W}$-doped $\mathrm{VO}_{2}$ films studied by photoelectron spectroscopy," Sol. Energ. Mat. Sol. Cell. 91, 1831-1835 (2007) [doi:10.1016/j.solmat.2007.06.013].

[7] S. Biermann, A. Poteryaev, A. I. Lichtenstein, and A. Georges, "Dynamical singlets and correlation-assisted Peierls transition in $\mathrm{VO}_{2}$, Phys. Rev. Lett. 94, 026404 (2005) [doi:10.1103/PhysRevLett.94.026404].

[8] H. Kim, B. Chae, D. Youn, S. Maeng, G. Kim, K. Kang, and Y. Lim, "Mechanism and observation of Mott transition in $\mathrm{VO}_{2}$-based two- and three-terminal devices," New J. Phys. 6, 52 (2004) [doi:10.1088/1367-2630/6/1/052].

[9] B. J. Powell and R. H. McKenzie, "Strong electronic correlations in superconducting organic transfer salts," J. Phys.: Condens. Matter 18, R827-R866 (2006) [doi:10.1088/0953-8984/18/45/R03].

[10] G. S. Kotliar, Y. Savrasov, K. Haule, V. S. Oudovenko, O. Parcollet, and C. A. Marianetti, "Electronic structure calculations with dynamical mean-field theory," Rev. Mod. Phys. 78, 865-951 (2006) [doi:10.1103/RevModPhys.78.865].

[11] H. A. Veleur, A. S. Barker, and C. N. Berglund, "Optical properties of $\mathrm{VO}_{2}$ between $0.25 \mathrm{eV}$ and $5 \mathrm{eV}$," Phys. Rev. 17, 788-798 (1968) [doi:10.1103/PhysRev.172.788].

[12] J. B. MacChesney and H. J. Guggenheim, "Growth and electrical properties of vanadium dioxide containing selected impurity ions," J. Phys. Chem. Solids 30, 225234 (1969) [doi:10.1016/0022-3697(69)90303-5].

[13] P. B. Allen, R. M. Wentzcovitch, and W. W. Schultz, "Resistivity of the hightemperature metallic phase of $\mathrm{VO}_{2}, "$ Phys. Rev. B 48, 4359-4363 (1993) [doi:10.1103/PhysRevB.48.4359].

[14] G. A. Niklasson and C. G. Granqvist, "Optical properties and solar selectivity of coevaporated Co- $\mathrm{Al}_{2} \mathrm{O}_{3}$ composite films," J. Appl. Phys. 55, 3382-3410 (1984) [doi:10.1063/1.333386].

[15] D. A. G. Bruggeman, "Berechnung verschiedener physikalischer Konstanten von Substanzen. I. Dielektrizitätskonstanten und Leitfähgkeiten der Mischkörper aus isotropen Substanzen,"Ann. Phys. Lpz. 24, 636-679 (1935) [doi:10.1002/andp.19354160705]. 
[16] G. B. Smith, A. I. Maaroof, and M. Cortie, "Percolation in nanoporous gold and the Principle of Universality from two dimensions to hyperdimensions," Phys Rev. B. 78,165418 (2008). [doi:10.1103/PhysRevB.78.165418]

[17] G. B. Smith, A. R. Gentle, and A. I. Maaroof, "Metal-insulator nanocomposites which act optically like homogeneous conductors," J. Nanophoton. 1, 013507 (2007) [doi:10.1117/1.2711706].

[18] A. I. Maaroof and B. L. Evans, "Onset of electrical conduction in Pt and Ni films," $J$. Appl. Phys. 76, 1047-1054 (1994) [doi:10.1063/1.357822].

[19] J. P. Clerc, G. Giraud, J. M. Laugier, and J. M. Luck, "The electrical conductivity of binary disordered systems, percolation clusters, fractals and related models," Adv. Phys. 39, 191-309 (1990) [doi:10.1080/00018739000101501].

[20] D. Stauffer and A. Aharony, Introduction to Percolation Theory, $2^{\text {nd }}$ ed., Taylor and Francis, London and Philadelphia (1992).

[21] J. Wu and D. S. McLachlan, "Percolation exponents and thresholds obtained from the nearly ideal continuum percolation system graphite-boron nitride," Phys. Rev. B 56, 1236-1248 (1997) [doi:10.1103/PhysRevB.56.1236].

[22] D. S. McLachlan, "An equation for the conductivity of binary mixtures with anisotropic grain structures," J. Phys. C 20, 865-877 (1987) [doi: 10.1088/0022-3719/20/7/004].

[23] J. Dai, X. Wang, Y. Huang, and X. Yi, "Modelling of temperature-dependent resistance in micro- and nanocrystalline $\mathrm{VO}_{2}$ thin films with random resistor networks," Opt. Eng. 47, 033801 (2008) [doi:10.1117/1.2894146]. 